УДК 517.518.5

\title{
On Invariant Estimates for Oscillatory Integrals with Polynomial Phase
}

\author{
Akbar R.Safarov* \\ Samarkand State University \\ Universitetsky boulevard, 15 \\ 140104, Samarkand \\ Uzbekistan
}

Received 17.04.2015, received in revised form 10.11.2015, accepted 21.12.2015

\begin{abstract}
In this paper we consider estimates for trigonometric (oscillatory) integrals with polynomial phase function of degree three. The main result of the paper is the theorem on uniform invariant estimates for trigonometric integrals. This estimate improves results obtained in the paper by D. A. Popov [1] for the case when the phase function is a sum of a homogeneous polynomial of third degree and a linear function, as well as the estimates of the paper [2] for the fundamental solution to the dispersion equation of third order.
\end{abstract}

Keywords: oscillatory integral, phase function, amplitude, invariant, discriminant. DOI: 10.17516/1997-1397-2016-9-1-102-107.

\section{Introduction and preliminaries}

Many problems of harmonic analysis, analytic number theory and mathematical physics are related to trigonometric (oscillatory) integrals with polynomial phase. Note that the form of such oscillatory integrals does not change under linear changes of variables, i.e. it is invariant under linear changes of variables. V.P. Palamodov posed the problem of estimating the trigonometric integral in terms of the coefficients of the phase function [3].

In this paper we give a solution of Palamodov's problem when the phase function is a sum of a homogeneous polynomial of third degree and linear terms. Such kind of integrals has been considered in the paper [2] in relation to the fundamental solution to third order dispersion equations. However, in that paper the estimates were obtained for the fundamental solutions for fixed coefficients of the principal part. It is an interesting problem to investigate the behavior of corresponding oscillatory integrals that depends on the coefficients of the polynomial, as proposed by V.P. Palamodov. Some invariant estimates were obtained by using invariants of classical groups in the paper [3]. However, these estimates are not optimal when discriminant of $P_{3}$ is small and the coefficients are large. In this paper we obtain optimal invariant estimates for trigonometric integrals (see Theorem 2.1).

There is a well-known asymptotic expansion for trigonometric integrals with smooth amplitude function as coefficients of the phase tend to infinity along a fixed direction, say $\lambda A$, where $A \in S^{5}$ is a fixed point on the unit sphere centered at the origin and $\lambda$ is a positive parameter. However, the behavior of a trigonometric integral may change significantly even if the vector $A$ varies a little. Thus, we come to the problem of combined estimates for trigonometric integrals,

*safarov-akbar@mail.ru

(C) Siberian Federal University. All rights reserved 
i.e. uniform estimates for trigonometric integrals depending on both large $\lambda$ and "small" parameters $A$, that has been investigated by many authors (see [4] for discussion and more references).

We obtain an estimate assuming that the amplitude belongs to some Sobolev space, so the coefficient in the estimate depends only on the norm of the amplitude function. The main estimate obtained in this paper agrees with the principal part of the asymptotic expansions for trigonometric integrals with the phase whose coefficients are of the form $\lambda A$ for a fixed vector $A \in S^{5}$. Depending on the vector $A$, the asymptotic behavior as $\lambda \rightarrow+\infty$ can have the form $O\left(\lambda^{-1 / 3}\right)\left(A_{2 \infty}\right.$ degenerated into the Airy-type singularity), $O\left(\lambda^{-1 / 2}\right)\left(D_{\infty}\right.$-type singularity), $O\left(\lambda^{-2 / 3}\right)\left(D_{4}^{ \pm}\right.$-type singularity), $O\left(\lambda^{-3 / 4}\right)$ ( $A_{3}$-type singularity), $O\left(\lambda^{-5 / 6}\right)\left(A_{2}\right.$-type singularity), $O\left(\lambda^{-1}\right)\left(A_{1}\right.$-type singularity).

In all cases our uniform estimate coincides with the principal part of the asymptotic expansion of oscillatory integrals with phase without linear terms and with smooth amplitude functions up to a constant. The results improve the estimates obtained in the paper [1] by D. A. Popov for particular case when the phase function is a polynomial, also it gives bounds for the fundamental solution to the dispersion equations in terms of its coefficients.

\section{Formulations of the main results}

Definition. The integral over $\mathbb{R}^{n}$

$$
J(\mathbb{P}, \varphi)=\int_{\mathbb{R}^{n}} e^{i \mathbb{P}(x, a)} \varphi(x) d x,
$$

where $\mathbb{P}: \mathbb{R}^{n} \times \mathbb{R}^{m} \rightarrow \mathbb{R}$ is a smooth real-valued function and $\varphi: \mathbb{R}^{n} \rightarrow \mathbb{C}$ is an element of the space of amplitude functions, is called an oscillatory integral with the phase $\mathbb{P}$ and the amplitude $\varphi$. In general the integral (1) does not converge in the Lebesgue sense. But it converges in the sense of distributions (see [5]).

We denote by $W_{1}^{n}\left(\mathbb{R}^{n}\right)$ the Sobolev space with the norm given by

$$
\|\varphi\|_{W_{1}^{n}\left(\mathbb{R}^{n}\right)}=\int_{\mathbb{R}^{n}}\left(\sum_{|\alpha| \leqslant n}\left|D^{\alpha} \varphi\right|\right) d x,
$$

where $D^{\alpha} \varphi$ is the derivative of $\varphi$ in the sense of distributions. Also, it can be considered as the completion of the Schwartz class of functions with respect to the norm $W_{1}^{n}\left(\mathbb{R}^{n}\right)$.

Let $\mathbb{P}$ be a polynomial function of the form

$$
\mathbb{P}=P_{3}+P_{1},
$$

where $P_{3}(x, y)=a_{0} x^{3}+3 a_{1} x^{2} y+3 a_{2} x y^{2}+a_{3} y^{3}$ and $P_{1}=c_{0} x+c_{1} y$.

We consider the following integral

$$
I=\int_{\mathbb{R}^{2}} \varphi(x, y) e^{i \mathbb{P}(x, y)} d x d y,
$$

where $\varphi \in W_{1}^{2}\left(\mathbb{R}^{2}\right)$. Obviously, this integral converges in the usual (Lebesgue) sense.

Theorem 2.1. Suppose $\varphi \in W_{1}^{2}\left(\mathbb{R}^{2}\right)$, then there exists a positive number $C$ such that the following estimate holds

$$
|I| \leqslant \frac{C\|\varphi\|_{W_{1}^{2}\left(\mathbb{R}^{2}\right)}}{|D|^{\frac{1}{6}}}
$$


where $D=3 a_{1}^{2} a_{2}^{2}+6 a_{0} a_{1} a_{2} a_{3}-4 a_{0} a_{2}^{3}-4 a_{1}^{3} a_{3}-a_{0}^{2} a_{3}^{2}$ is the discriminant of the polynomial $P_{3}(x, y)$.

Remark 1. If $c_{0}=c_{1}=0$ then theorem 4 of the paper [3] yields

$$
|J| \leqslant \frac{C\|\varphi\|_{W_{1}^{2}\left(\mathbb{R}^{2}\right)}}{N^{\frac{1}{6}}+|M|^{\frac{1}{4}}+\frac{|D|^{\frac{2}{3}}}{N}},
$$

where $N=a_{0}^{2}+3 a_{1}^{2}+3 a_{2}^{2}+a_{3}^{2}, M=a_{1}^{2}+a_{2}^{2}-a_{0} a_{2}-a_{1} a_{3}$.

So, when $\frac{|D|^{\frac{2}{3}}}{N}$ is the dominant term then our estimate does not contain the factor $N$. It is obvious that our estimates are much better when $\frac{D}{N^{2}}$ is small and $N \gg 1$. The factor $N$ appears in the estimate for the Sobolev norm of a function. But this norm is not invariant under a linear change of variables.

We use another technique based on estimates for oscillatory integrals over a triangle (with a discontinuous amplitude). It allows us to obtain a more precise estimate (see Th.4.1).

Remark 2. By using Popov's [1] Theorem 4.1 one can obtain the estimate (in our notation)

$$
|J| \leqslant C_{\varepsilon} N^{\frac{1}{3}(11+\varepsilon)} D^{-2},
$$

where $0<\varepsilon \leqslant 1$ and $C_{\varepsilon}$ is a constant. Note that $N^{\frac{11}{3}} D^{-2}>N D^{-\frac{2}{3}}$, hence our estimate also improves the bound given by Popov.

From theorem 2.1 and theorem 4 of the paper [3] we have the following

Theorem 2.2. For the integral (2) the following estimate holds

$$
|I| \leqslant \frac{C\|\varphi\|_{W_{1}^{2}\left(\mathbb{R}^{2}\right)}}{|D|^{\frac{1}{6}}+|M|^{\frac{1}{4}}+N^{\frac{1}{6}}},
$$

where $N=a_{0}^{2}+3 a_{1}^{2}+3 a_{2}^{2}+a_{3}^{2}, M=a_{1}^{2}+a_{2}^{2}-a_{0} a_{2}-a_{1} a_{3}$.

\section{Proof of the main results}

First we prove Theorem 2.1. Assuming that $|D| \geqslant M$ (where $M$ is a fixed sufficiently large positive number), possibly after a rotation, we may take $\left|a_{0}\right|=\max \left\{\left|a_{0}\right|,\left|a_{1}\right|,\left|a_{2}\right|,\left|a_{3}\right|\right\}$. The estimate (6) is trivial when $|D|<M_{0}$. Note that the Schwartz class of functions is dense in $W_{1}^{2}\left(\mathbb{R}^{2}\right)$. Hence, it is enough to prove the estimate (6) for $\varphi$ from the Schwartz space. Integrating by parts, we get

$$
I=\int_{-\infty}^{\infty} \int_{-\infty}^{\infty} \frac{\partial^{2} \varphi}{\partial x \partial y}\left(\int_{-\infty}^{x} \int_{-\infty}^{y} e^{i \mathbb{P}(\xi, \eta)} d \eta d \xi\right) d x d y
$$

From this, the inequality

$$
|I| \leqslant \sup _{x, y}\left|\int_{-\infty}^{x} \int_{-\infty}^{y} e^{i \mathbb{P}(\xi, \eta)} d \xi d \eta\right| \int_{\mathbb{R}^{2}}\left|\frac{\partial^{2} \varphi}{\partial x \partial y}\right| d x d y
$$

follows trivially.

Now, we estimate the following integral

$$
\int_{-\infty}^{x} \int_{-\infty}^{y} e^{i \mathbb{P}(\xi, \eta)} d \xi d \eta
$$


First, we change variables in the phase function. To be precise, we assume that the equation $P_{3}(x, y)=0$ has only one real root. We can represent the principal part of the phase function as

$$
P_{3}(x, y)=a_{0}\left(x-\alpha_{1} y\right)\left(x-\alpha_{2} y\right)\left(x-\alpha_{3} y\right)
$$

where $\alpha_{1}, \alpha_{2}, \alpha_{3}$ are the roots of $P_{3}$.

Suppose $\alpha_{3}$ is the real root of $P_{3}(x, 1)=0$, then $\alpha_{1}=\bar{\alpha}_{2}$. We use the following change of variables (cf. [6], also [1]):

$$
\begin{gathered}
x=\frac{2\left(\alpha_{1}-\alpha_{3}\right)\left(\alpha_{2}-\alpha_{3}\right)+\left(\alpha_{1}+\alpha_{2}-2 \alpha_{3}\right) \alpha_{3}}{2\left(\alpha_{1}-\alpha_{3}\right)\left(\alpha_{2}-\alpha_{3}\right)\left|\alpha_{1}-\alpha_{2}\right|} x_{1}+\frac{\alpha_{3}}{2\left(\alpha_{1}-\alpha_{3}\right)\left(\alpha_{2}-\alpha_{3}\right)} y_{1}, \\
y=\frac{\alpha_{1}+\alpha_{2}-2 \alpha_{3}}{2\left(\alpha_{1}-\alpha_{3}\right)\left(\alpha_{2}-\alpha_{3}\right)\left|\alpha_{1}-\alpha_{2}\right|} x_{1}+\frac{1}{2\left(\alpha_{1}-\alpha_{3}\right)\left(\alpha_{2}-\alpha_{3}\right)} y_{1} .
\end{gathered}
$$

Then we have

$$
\mathbb{P}=\frac{a_{0}}{4\left(\alpha_{1}-\alpha_{3}\right)\left(\alpha_{2}-\alpha_{3}\right)\left|\alpha_{1}-\alpha_{2}\right|} x_{1}\left(y_{1}^{2}-\operatorname{sgn}\left(\alpha_{1}-\alpha_{2}\right)^{2} x_{1}^{2}\right) .
$$

Where $\operatorname{sgn}\left(\alpha_{1}-\alpha_{2}\right)^{2}=1$ if both $\alpha_{1}, \alpha_{2}$ are distinct real numbers and $\operatorname{sgn}\left(\alpha_{1}-\alpha_{2}\right)^{2}=-1$ if $\alpha_{1}, \alpha_{2}$ are non-real complex conjugate numbers.

We know that the function (6) has singularities of type $D_{4}^{ \pm}\left(\right.$depending on $\left.\operatorname{sgn}\left(\alpha_{1}-\alpha_{2}\right)^{2}\right)$, when $\alpha_{1}, \alpha_{2}$ are complex numbers then surely $\alpha_{1}=\bar{\alpha}_{2}$. Note that the change of variables $(5)$ can be used in the case when $P_{3}(x, 1)$ has three real roots(compare with [1]). After the change of variables, $[x, \infty) \times[y, \infty)$ transforms into a triangle. Further on, by a triangle we mean any proper triangle, any angle in the plane, or a strip.

Now we consider the integral

$$
J_{T}=\int_{T} e^{i \lambda\left(\xi^{3} \pm \xi \eta^{2}+s_{1} \xi+s_{2} \eta\right)} d \xi d \eta
$$

where $T$ is a triangle.

Theorem 3.1. There exists a constant $C$ such that for any $\left(s_{1}, s_{2}\right) \in \mathbb{R}^{2}$ and $|\lambda| \geqslant 1$ the following estimate

$$
\left|J_{T}\right| \leqslant \frac{C}{\lambda^{\frac{2}{3}}}
$$

holds true.

Theorem 3.1 is an analog of the more general theorem by J.|,Duistermaat [7] for the case $T=\mathbb{R}^{2}$. It is proved in the paper [9] (see also [8]).

Without loss of generality we may assume that $\frac{a_{0}}{4\left|\left(\alpha_{1}-\alpha_{3}\right)\left(\alpha_{2}-\alpha_{3}\right)\left(\alpha_{1}-\alpha_{2}\right)\right|} \geqslant 1$. From Theorem 3.1 we have the following estimates

$$
\left|\int_{T} e^{i \mathbb{P}(\xi, \eta)} d \xi d \eta\right| \leqslant \frac{\frac{C}{2\left|\alpha_{1}-\alpha_{3}\right|\left|\alpha_{2}-\alpha_{3}\right|\left|\alpha_{1}-\alpha_{2}\right|}}{\left|\frac{a_{0}}{4\left(\alpha_{1}-\alpha_{3}\right)\left(\alpha_{2}-\alpha_{3}\right)\left|\alpha_{1}-\alpha_{2}\right|}\right|^{\frac{2}{3}}}=\frac{C}{\left|a_{0}^{4}\left(\alpha_{1}-\alpha_{3}\right)^{2}\left(\alpha_{2}-\alpha_{3}\right)^{2}\left(\alpha_{1}-\alpha_{2}\right)^{2}\right|^{\frac{1}{6}}} .
$$

Note that $D=\frac{a_{0}^{4}\left(\alpha_{1}-\alpha_{3}\right)^{2}\left(\alpha_{2}-\alpha_{3}\right)^{2}\left(\alpha_{1}-\alpha_{2}\right)^{2}}{27}$ (see [1]). 
So, for any element $\varphi$ of the Schwartz space the following estimate holds

$$
|I| \leqslant \frac{C\|\varphi\|_{W_{1}^{2}\left(\mathbb{R}^{2}\right)}}{|D|^{\frac{1}{6}}} .
$$

Since the set of infinitely differentiable rapidly decreasing functions is dense in $W_{1}^{2}\left(\mathbb{R}^{2}\right)$, we obtain a required bound by passing to the limit. This completes the proof of Theorem 2.1.

Now we shall give some auxiliary results.

Proposition. There exists a constant $C$ such that for the integral (1) the following estimate

$$
|J| \leqslant \frac{C\|\varphi\|_{W_{1}^{1}\left(\mathbb{R}^{2}\right)}}{|N|^{\frac{1}{6}}+|M|^{\frac{1}{4}}}
$$

holds true.

Proof. The proof, in fact, follows from the following results of the paper [3].

Let $\varphi$ belong to the Sobolev space $W_{1}^{2}\left(\mathbb{R}^{2}\right)$ and

$$
p(x, y)=p_{n}(x, y)+p_{n-1}(x, y)+\ldots+p_{1}(x, y)
$$

where $p_{k}$ is a homogeneous polynomial of degree $k$. Consider a trigonometric integral of the form

$$
J=\int_{\mathbb{R}^{2}} \varphi(x, y) e^{i p(x, y)} d x d y
$$

Lemma 2.4. The integral (8) is estimated as follows

$$
|J| \leqslant \frac{k\|\varphi\|_{W_{1}^{1}\left(\mathbb{R}^{2}\right)}}{\left|d^{n} p\right|^{\frac{1}{n}}} .
$$

Let $E_{n}$ be the space of binary forms of degree $n$. The group $S L(2, \mathbb{R})$ acts on it. If $D$ is an invariant of the group $S L(2, \mathbb{R})$, its value at the form $p$ will be denoted $D(p)$.

The space of polynomials of degree at most $n$ is injectively imbedded in $E_{n}$. Indeed, it suffices to multiply each term by a power of a new variable such that the result is a homogeneous form of degree $n$. This is an isomorphic mapping from the space of polynomials of degree at most $n$ into the space $E_{n}$. We shall denote it by $\Phi_{n}$. Thus, for a polynomial $q$ in one variable we can determine the value of the invariant $D$ of the group $S L(2, \mathbb{R})$ from the formula

$$
\widetilde{D}(q)=D\left(\Phi_{n}(q)\right)
$$

Lemma 2.5 Let $D$ be a homogeneous invariant of degree $m$ in $E_{n-1}$. Then for the integral (8) the following inequality holds

$$
|J| \leqslant \frac{k\|\varphi\|_{W\left(\mathbb{R}^{2}\right)}}{\left(\left|D\left(\frac{\partial p_{n}}{\partial x}\right)\right|+\left|D\left(\frac{\partial p_{n}}{\partial y}\right)\right|\right)^{\frac{2}{m(n+1)}}} .
$$

Finally, the proof of Theorem 2.2 follows from Theorem 2.1 and Proposition.

Acknowledgment. The author is grateful to Professor I. A. Ikromov for suggesting the problem and constant attention to my work and to the referee for many valuable remarks.

This work is supported by the grant of the Republic of Uzbekistan, grant F-4-17. 


\section{References}

[1] D.A.Popov, Note on combained uniform estimates for oscillating integrals with simple singularyties, Izv. RAN., 72(2008), no. 4, 173-196 in (Russian).

[2] M.Ben-Artzi, H.Koch, J.-C. Saut, Dispersion Estimates for third order equations in two dimensions, Comm. Partial Differential Equations, 28(2003), no.11-12, 1943-1974.

[3] I.A.Ikromov, Invariant estimates of two-dimentional trigonometrical integrals, Matem. Sb. USSR, 67(1990), no. 2, 473-488.

[4] D.A.Popov, Estimates with constants for some classes of oscillatory integrals, Russian Math. Surveys, 52(1997), no. 1, 73-145.

[5] L.Hörmander, The analysis of linear partial differential operators. I, Distribution theory and Fourier analysis, Grundlehren der Mathematichen Wissenschaften, vol. 256, Springer-Verlag, Berlin, 1983.

[6] G.B.Gurevich, Foundations of the theory of algebraic invariants, Noordhoff, 1964.

[7] J.Duistermaat, Oscillatory integrals Lagrange immersions and unifoldings of singularities, Comm. Pure. Appl. Math., 27(1974), no. 2, 207-281.

[8] A.Safarov, On uniform estimates for model trigonometrical integrals with discontinuous amplitude, Uzbek. Mat. Zhurnal, (2015) (in Russian).

[9] S.Aronhold, Zur Theorie der homogenen Functionen dritten Grades von drei Variabeln, $J$. Reine Angew. Math., 39(1850), 140-159.

[10] S.N.Bernstein, Extrenal properties of polynomials, ONTI, Moscow, 1937 (in Russian).

[11] Clebsch, Gordan, Uber cubische-ternäre Formen, Mathematische Annalen, 6(1873), 436512 .

[12] V.P.Palamodov, Deformations of Hopf manifolds and the Poincaré-Dulac theorem, Funktional. Anal. Appl., 17(1983), no. 4, 252-259.

[13] I.A.Ikromov, A.Safarov, Invariant estimates for oscillatory integral with homogeneous polynomial, Vestnik Bashkirskogo Universiteta, 19(2014), no. 3 ,774-779 (in Russian).

\section{Об инвариантных оценках осциллирующих интегралов с полиномиальной фазой}

\section{Акбар Р. Сафаров}

В этой статье мы рассмотрим инвариантные оценки тригонометрических (осциллирующих) интегралов с полиномиальной фазой. Основным результатом является теорема о равномерной инвариантной оченке тригонометрического интеграла. Полученная оценка улучшает результаты работы Д.А.Попова [1] для случая, когда фазовая функиия является суммой однородного полинома третьей степени и линейной функиии, а также оценки работы [2] для фундаментального решения дисперсионного уравнения третъей степени.

Ключевые слова: осциллирующий интеграл, фазовая функция, амплитуда, инвариант, дискриминант. 